\title{
Association Between Polymorphisms of the Complement 3 Gene and Schizophrenia in a Han Chinese Population
}

\author{
Shangchao Zhang ${ }^{\mathrm{a}}$ Na Zhou ${ }^{\mathrm{b}}$ Rui Liu ${ }^{\mathrm{a}}$ Wenwang Rao ${ }^{\mathrm{a}}$ Mingjia Yang ${ }^{\mathrm{a}}$ \\ Bonan Cao ${ }^{a}$ Guojun Kang ${ }^{a}$ Qi Kang ${ }^{a}$ Xiaojing Zhu ${ }^{a}$ Rixin Lia Yaqin Yu ${ }^{a}$ \\ Qiong $\mathrm{Yu}^{\mathrm{a}}$
}

aDepartment of Epidemiology and Biostatistics, School of Public Health, Jilin University, Changchun, bDepartment of Pharmacy, Hospital of Stomatology, Jilin University, Changchun, China

\section{Key Words}

Schizophrenia • Complement 3 gene $\cdot$ Single nucleotide polymorphisms (SNPs)

\begin{abstract}
Background/Aims: Schizophrenia is a severe psychiatric disorder, and complement 3 (C3) is closely related to schizophrenia. We investigated the association between C3 polymorphisms and schizophrenia in a Northeast Han Chinese population. Methods: A total of 2240 Chinese people, consisting of 1086 patients with schizophrenia and 1154 healthy controls, were recruited for this study. Ten single nucleotide polymorphisms (SNPs; rs11569562, rs344555, rs2241393, rs2241392, rs11569514, rs445750, rs451760, rs11672613, rs2230205, and rs2250656) in C3 were selected and genotyped. Results: Genotype distribution analysis indicated that rs11569514 was significantly associated with schizophrenia. In the dominant model (AA vs. GG+GA genotypes), we found a significant protective effect for rs344555 against schizophrenia (odds ratio [OR]: $0.72,95 \%$ confidence interval [CI]: $0.53-0.99, P=0.04$ ). In the codominant model (TT vs. AA), we found a significant risk effect for rs 11569514 on schizophrenia (OR: 4.39, 95\% CI: 2.06-9.37, $P<0.001$ ). Haplotypes, including TG (rs11569562 and rs344555), TGG (rs11569562-rs344555rs2241393), AG (rs344555-rs2241393), CGGGT (rs11569562-rs344555-rs2241393-rs2241392rs11569514), and ACGTG (rs11569514-rs445750-rs451760-rs11672613-rs2230205), showed either a risk or protective role for schizophrenia. Conclusions: SNP rs11569514 in C3 and haplotypes of C3 variants were associated with schizophrenia in a Han Chinese population.

\section{Introduction}

Schizophrenia (SCZ) is a severe mental disorder that affects approximately $0.5-1 \%$ of the population worldwide and has a profound impact on society and the economy $[1,2]$. SCZ is highly prevalent among young adults [3], and its incidence rate is slightly higher in males than in females (1.4:1) [4]. Compared with the general population, patients with SCZ \begin{tabular}{ll}
\hline Qiong Yu & Department of Epidemiology and Biostatistics, School of Public Health, Jilin University \\
& 1163 Xinmin Street, Changchun 130021 (China)
\end{tabular} Tel. +86 431 85619451, Fax +86 431 85645486, E-Mail yuqiong@jlu.edu.cn 
have a shortened life span [5] and experience a wide variety of symptoms, including serious depression, substance abuse, obsessive-compulsive behavior, cognitive learning deficits, and memory problems $[6,7]$.

The etiology and pathogenesis of SCZ are still not understood clearly. Numerous studies have shown that SCZ is caused by a combination of multiple risk factors, which can be broadly classified into genetic factors (intrinsic factors) and environmental factors (external factors). Therefore, SCZ is the result of an interaction between genes and the environment $[8,9]$. The importance of genetic components as well as developmental and environmental influences in SCZ has been demonstrated by family, twin, and adoption studies [10-12].

Recently, a large-scale genome-wide association study demonstrated that multiple genetic risk factors contribute to the occurrence of SCZ [13]. While previous studies have emphasized the polygenic nature of SCZ, it is a challenge to provide a comprehensive explanation linking genetic associations with the underlying pathophysiology.

SCZ has high heritability (approximately 60-80\%) [14]. Over the past few decades, variants in several candidate genes, including DRD2, ZNF804A, CHGB, NRGN, SHANK3, BACE1, $F G A$, and complement 3 (C3), were found to be associated with SCZ [15-20]. Particularly, the concentration of $C 3$ in the blood of patients with SCZ has been extensively studied, and the role of complement in SCZ is beginning to be more widely explored. Several studies have shown that the level of $C 3$ is higher in the blood of SCZ patients than in healthy subjects, indicating that the $C 3$ fast (F) allotype may be a potential biomarker for the diagnosis of SCZ [21].

Although C3 may play a potential role in SCZ, the pathophysiology of SCZ remains unknown [22]. Due to the importance of $C 3$ in multiple immunoregulatory processes, the investigation of $C 3$ polymorphisms remains of interest in SCZ research. However, a handful of genetic studies have been performed to study the association between single nucleotide polymorphisms (SNPs) in C3 and SCZ, but no significant association has been discovered [16]. In this study, we selected 10 SNPs of $C 3$ (rs11569562, rs344555, rs2241393, rs2241392, rs11569514, rs445750, rs451760, rs11672613, rs2230205, and rs2250656) to examine the association between $C 3$ variation and SCZ.

\section{Materials and Methods}

\section{Participants}

This study was approved by the Ethics Committee of the School of Public Health, Jilin University. All participants provided written informed consent before enrolling in the study.

A total of 1086 patients with SCZ were enrolled from the Sixth Hospital of Changchun City and the Encephalopathy Hospital of Jilin Province, from 2006 to 2012. All participants belonged to the Han population in China. Each patient was diagnosed by two or more physicians based on the International Classification of Diseases 10 criteria for SCZ, and in the meantime, referred to the Chinese Psychiatric Classification and Diagnostic Criteria Program, Third Edition.

Control subjects were 1154 healthy Han Chinese volunteers enrolled from the First Hospital of Jilin University in Changchun City, Jilin Province, China. Before sampling, all control subjects were physically healthy and had no family history of mental illness. Those with a medical illness or drug and alcohol abuse/ dependence were excluded.

\section{SNP selection and genotyping}

Based on previous studies, we selected 10 tag SNPs (rs11569562, rs344555, rs2241393, rs2241392, rs11569514, rs445750, rs451760, rs11672613, rs2230205, and rs2250656) in C3 using the Haploview program (http://hapmap.ncbi.nlm.nih.gov/) [23]. The threshold for the minor allele frequency of these 10 tag SNPs was set at greater than 0.1 in CHB with $\mathrm{r}^{2}>0.8$.

We collected a $5-\mathrm{mL}$ blood sample from each subject and stored the specimen at $-20^{\circ} \mathrm{C}$ in an anticoagulant Plexiglas tube. For genotyping these 10 SNPs, we utilized a commercial DNA extraction kit 
(ClotBlood DNA kit, Catalog number: CW0565, Beijing, China) and extracted genomic DNA from peripheral blood lymphocytes, according to the manufacturer's instructions. Assay Design software 3.1 (Sequenom, Inc., San Diego, CA) was used to design PCR primers for each SNP (Table 1). Genomic DNA amplification was conducted using specific forward and reverse primer pairs. HotStarTaq Master Mix Kit (Qiagen, Hilden, Germany) was used for PCR. PCR amplification conditions were set for an initial cycle at $94^{\circ} \mathrm{C}$ for 15 min, 45 cycles at $94^{\circ} \mathrm{C}$ for $20 \mathrm{~s}, 56^{\circ} \mathrm{C}$ for $30 \mathrm{~s}$, and $72^{\circ} \mathrm{C}$ for $60 \mathrm{~s}$, and finally $72^{\circ} \mathrm{C}$ for $3 \mathrm{~min}$. PCR products were subjected to the shrimp alkaline phosphatase reaction and single-base extension. After desalting, the PCR products were analyzed using the MassARRAY system (Sequenom, Inc.) for SNP genotyping.

\section{Statistical analysis}

Statistical software SPSS 24.0 (SPSS Inc., Chicago, IL) was used to analyze the data. The $\chi^{2}$ test or Student's t-test was used to compare sex and age differences, respectively, between the SCZ and control groups. The Hardy-Weinberg disequilibrium test was applied to evaluate each SNP in both groups. Odds ratios (ORs) and 95\% confidence intervals (CIs) for SNPs in C3 associated with SCZ were tested with logistic regression models adjusted for age and gender. Quanto 1.2.4 software was used to calculate the statistical power of the sample. The Akaike information criterion was used to select the best genetic model for each SNP. The SNPStats program (http://bioinfo.iconcologia.net/SNPStats) [24] was applied to carry out haplotype analysis: the most frequent haplotype was regarded as the reference group. A $P$-value of 0.05 or less was used to determine statistical significance, and all statistical tests were twosided.
Table 1. PCR primer sequences for each SNP. F, forward; R, reverse

\begin{tabular}{ll}
\hline SNP & \multicolumn{1}{c}{ Primer sequence $\left(5^{\prime}-3^{\prime}\right)$} \\
\hline rs11569562 & F: ACGTTGGATGTACTATGCAATGAGAGTGAC \\
& R: ACGTTGGATGTGACTGTAGTTTTCGTGGC \\
rs344555 & F: ACGTTGGATGTCTGAGCAAGCCACACTTAC \\
& R: ACGTTGGATGCTTGGTATCCCTGGGTATG \\
rs2241393 & F: ACGTTGGATGGAACCTACTAGAGAGTGCAG \\
& R: ACGTTGGATGTTCTGTCTCTCGGAGAGTTC \\
rs2241392 & F: ACGTTGGATGGCTCAACACACAGCTTGGAG \\
& R: ACGTTGGATGGCAGTGGAGTTGAGAATCAG \\
rs11569514 & F: ACGTTGGATGGAGGGTTTTCAGCCCATTTC \\
& R: ACGTTGGATGCGCAACTGTACTGATCATGT \\
rs445750 & F: ACGTTGGATGTTCTGTTGAGCAGCCATGTC \\
& R: ACGTTGGATGGGCAACAAGAGCAAAACTCC \\
rs451760 & F: ACGTTGGATGCAGAGACAGACTCCATCTGA \\
& R: ACGTTGGATGATCTAAAGGTGTGACCGCTG \\
rs11672613 & F: ACGTTGGATGAAAGAGGCGATAGTGTGAGG \\
& R: ACGTTGGATGAAAAGTACAGCCCACCATCC \\
rs2230205 & F: ACGTTGGATGTCTTCTCAGCAGCCTTGGGT \\
& R: ACGTTGGATGGCGTGTTCGTGCTGAATAAG \\
rs2250656 & F: ACGTTGGATGTCCTTGCCTGCCCATTATTC \\
& R: ACGTTGGATGTCGCACCTCCTTCACATGCC \\
\hline
\end{tabular}

Table 2. Comparison of the allelic frequencies of 10 C3 tag SNPs between the cases and controls. ${ }^{*} P$-values were adjusted for age and gender

\begin{tabular}{lccccc}
\hline SNP & Allele & Case (\%) & Control (\%) & $\chi^{2}$ & $P^{*}$ \\
\hline rs11569562 & C & $1140(53.7)$ & $1193(51.9)$ & 1.665 & 0.197 \\
& T & $984(46.3)$ & $1105(48.1)$ & & \\
rs344555 & A & $626(29.6)$ & $621(27.0)$ & 3.115 & 0.078 \\
& G & $1490(70.4)$ & $1677(73.0)$ & & \\
rs2241393 & C & $751(35.2)$ & $850(36.9)$ & 1.579 & 0.209 \\
& G & $1385(64.8)$ & $1452(63.1)$ & & \\
rs2241392 & C & $527(25.3)$ & $578(25.3)$ & 0.012 & 0.912 \\
& G & $1553(74.7)$ & $1710(74.7)$ & & \\
rs11569514 & A & $1612(90.1)$ & $2082(91.2)$ & 1.463 & 0.226 \\
& T & $178(9.9)$ & $202(8.8)$ & & \\
rs445750 & C & $335(17.1)$ & $357(15.6)$ & 2.046 & 0.153 \\
& T & $1621(82.9)$ & $1931(84.4)$ & & \\
rs451760 & A & $1954(91.4)$ & $2114(92.3)$ & 1.699 & 0.192 \\
& G & $184(8.6)$ & $176(7.7)$ & & \\
rs11672613 & C & $867(41.3)$ & $951(41.4)$ & 0.012 & 0.914 \\
& T & $1231(58.7)$ & $1345(58.6)$ & & \\
rs2230205 & A & $1015(47.5)$ & $1086(47.2)$ & 0.009 & 0.925 \\
& G & $1123(52.5)$ & $1214(52.8)$ & & \\
rs2250656 & A & $1683(78.6)$ & $1833(79.6)$ & 0.414 & 0.520 \\
& G & $457(21.4)$ & $471(20.4)$ & & \\
\hline & & & & &
\end{tabular}

\section{Results}

The average age of the SCZ patients and controls was $34.24 \pm 12.26$ years and 36.47 \pm 10.93 years, respectively; the cases were significantly younger than the controls $(P<$ $0.001)$. There was a statistically significant sex difference between the cases and controls $\left(\chi^{2}=8.733, P=0.003\right)$. In the Hardy-Weinberg equilibrium (HWE) test, we found that the genotype distributions of the 10 tag SNPs in both the SCZ group and control group were in accordance with the HWE. The genotype distributions of rs11569514 and rs11672613 revealed a deviation from the HWE in the patient group. We randomly selected 10\% of samples from the case group to check the genotypes of rs11569514 and rs11672613, and no genotyping errors were found.

We found no significant difference in the allele frequencies of the 10 tag SNPs of C3 between the SCZ patients and healthy controls in multivariable logistic regression models (Table 2). As shown in Table 3, the genotype frequencies of these 10 tag SNPs in C3 indicated that rs11569514 was significantly different between the cases and controls $(P=0.035)$, but there was no significant difference in the genotype distribution of the other 9 SNPs between 
the SCZ and control groups.

The inheritance model for rs11569562, rs344555, rs2241393, and rs2250656 was dominant, the inheritance model for rs2241392, rs11672613, and rs2230205 was overdominant, the inheritance model for rs445750 and rs451760 was recessive, and the inheritance model for rs11569514 was codominant (Table 4). In the dominant model (AA vs. GG+GA genotypes), we found a significantly protective effect for variant rs344555 on SCZ (OR: 0.72, 95\% CI: $0.53-0.99, P=0.040$ ). In the codominant model, we also found a significant association between rs11569514 and SCZ in the TT vs. AA genotype comparison (OR: 4.39, 95\% CI: 2.069.37, $P<0.0001$ ).

Polygenic diseases may be associated with a specific combination of alleles, rather than a single allele. To assess the combined effects of the SNPs in $C 3$ on SCZ, we performed haplotype analyses (Table 5). Haplotypes AG (rs344555rs2241393), CGGGT (rs11569562rs344555-rs2241393-rs2241392rs11569514), and ACGTG (rs11569514-rs445750-rs451760rs11672613-rs2230205) were associated with an increased risk for SCZ (OR: 1.29, 95\% CI: 1.00-1.66, $P=0.049$; OR: 1.85 , 95\% CI: $1.18-$ 2.91, $P=0.0073$; OR: $1.37,95 \% \mathrm{CI}$ : 1.01-1.85, $P=0.044$, respectively). Haplotypes TG (rs11569562rs344555) and TGG (rs11569562rs344555-rs2241393) were associated with SCZ (OR: 0.81, 95\% CI: $0.69-0.95, P=0.012$; OR: 0.76, 95\% CI: $0.60-0.96, P=0.019$, respectively). No other haplotype showed a significant association with SCZ.

We also calculated the power of these 10 tag SNPs with an OR ranging from 1.4 to $2.0(\alpha=$ 0.05 , two-tailed test) to detect genetic variance of complex traits using SNP data in unrelated samples. We detected a power of 0.99860.9999 for rs11569562, 0.9122-0.9999 for rs344555, 0.9829-0.9999 for rs2241393, 0.8490-0.9999 for rs2241392, 0.2085-0.9999 for rs11569514, 0.5085-0.9999 for rs445750, 0.1695-0.9999 for rs451760, 0.9948-0.9999 for rs11672613, 0.9987-0.9999 for rs2230205, and $0.7109-0.9999$ for rs2250656.
Table 3. Comparison of the genotype distributions of 10 C3 tag SNPs between the cases and controls. ${ }^{*} P$-values were adjusted for age and gender

\begin{tabular}{lccccc}
\hline SNP & Genotype & Case (\%) & Control (\%) & $\chi^{2}$ & $P^{*}$ \\
\hline rs11569562 & CC & $291(27.4)$ & $300(26.1)$ & 1.867 & 0.393 \\
& CT & $558(52.5)$ & $593(51.6)$ & & \\
& TT & $213(20.1)$ & $256(22.3)$ & & \\
rs344555 & AA & $103(9.7)$ & $80(7.0)$ & 4.438 & 0.109 \\
& GA & $420(39.7)$ & $461(40.1)$ & & \\
rs2241393 & GG & $535(50.6)$ & $608(52.9)$ & & \\
& CC & $130(12.2)$ & $163(14.2)$ & 1.997 & 0.368 \\
& CG & $491(46.0)$ & $524(45.5)$ & & \\
rs2241392 & GG & $447(41.8)$ & $464(40.3)$ & & \\
& CC & $67(6.4)$ & $74(6.5)$ & 0.034 & 0.983 \\
& CG & $393(37.8)$ & $430(37.6)$ & & \\
rs11569514 & GG & $580(55.8)$ & $640(55.9)$ & & \\
& AA & $748(83.5)$ & $949(83.1)$ & 17.969 & $<0.001$ \\
& TA & $116(13.0)$ & $184(16.1)$ & & \\
rs445750 & TT & $31(3.5)$ & $9(0.8)$ & & \\
& CC & $31(3.2)$ & $31(2.7)$ & 2.037 & 0.361 \\
& CT & $273(27.9)$ & $295(25.8)$ & & \\
rs451760 & TT & $674(68.9)$ & $818(71.5)$ & & \\
& AA & $896(83.8)$ & $974(85.1)$ & 3.322 & 0.190 \\
& GA & $162(15.2)$ & $166(14.5)$ & & \\
rs11672613 & GG & $11(1.0)$ & $5(0.4)$ & & \\
& CC & $158(15.1)$ & $193(16.8)$ & 2.556 & 0.279 \\
& CT & $551(52.5)$ & $565(49.2)$ & & \\
rs2230205 & TT & $340(32.4)$ & $390(34.0)$ & & \\
& AA & $232(21.7)$ & $257(22.4)$ & 0.994 & 0.608 \\
& AG & $551(51.5)$ & $572(49.7)$ & & \\
rs2250656 & GG & $286(26.8)$ & $321(27.9)$ & & \\
& AA & $658(61.5)$ & $723(62.8)$ & 0.481 & 0.786 \\
& AG & $367(34.3)$ & $387(33.6)$ & & \\
& GG & $45(4.2)$ & $42(3.6)$ & & \\
\hline & & & & &
\end{tabular}

Table 4. Logistic regression analysis of the association between the SNPs in the C3 gene with SCZ. *ORs, 95\% CIs, and $P$-values were adjusted for age and gender

\begin{tabular}{lcccccc}
\hline SNP & Genotype & Inheritance & Case (\%) & Control (\%) & OR (95\% CI)* & $P^{*}$ \\
\hline rs11569562 & TT & Dominant & $210(19.9)$ & $256(22.3)$ & 1.00 & 0.21 \\
& CC+CT & & $843(80.1)$ & $893(77.7)$ & $1.14(0.93-1.41)$ & \\
rs344555 & AA & Dominant & $102(9.7)$ & $80(7.0)$ & 1.00 & 0.04 \\
& GG+GA & & $946(90.3)$ & $1069(93)$ & $0.72(0.53-0.99)$ & \\
rs2241393 & CC & Dominant & $127(12.0)$ & $163(14.2)$ & 1.00 & 0.17 \\
& GG+CG & & $932(88.0)$ & $988(85.8)$ & $1.19(0.93-1.53)$ & \\
rs2241392 & GG+CC & Overdominant & $640(62.1)$ & $714(62.4)$ & 1.00 & 0.85 \\
& GG & & $391(37.9)$ & $430(37.6)$ & $1.02(0.85-1.21)$ & \\
rs11569514 & AA & Codominant & $739(83.5)$ & $949(83.1)$ & 1.00 & \\
& TA & & $116(13.1)$ & $184(16.1)$ & $0.81(0.63-1.04)$ & 0.101 \\
& TT & & $31(3.4)$ & $9(0.8)$ & $4.39(2.06-9.37)$ & $<0.0001$ \\
rs445750 & CT+CC & \multirow{2}{*}{ Recessive } & $302(31.2)$ & $326(28.5)$ & 1.00 & 0.16 \\
& TT & & $667(68.8)$ & $818(71.5)$ & $0.87(0.72-1.06)$ & \\
rs451760 & AA+GA & \multirow{2}{*}{ Recessive } & $1048(99.0)$ & $1140(99.6)$ & 1.00 & 0.075 \\
& GG & & $11(1.0)$ & $5(0.4)$ & $2.53(0.87-7.34)$ & \\
rs11672613 & TT+CC & Overdominant & $493(47.5)$ & $583(50.8)$ & 1.00 & 0.13 \\
& GT & & $546(52.5)$ & $565(49.2)$ & $1.14(0.96-1.35)$ & \\
rs2230205 & GG+AA & Overdominant & $514(48.5)$ & $578(50.3)$ & 1.00 & 0.32 \\
& AG & & $545(51.5)$ & $572(49.7)$ & $1.09(0.92-1.29)$ & \\
rs2250656 & GG & \multirow{2}{*}{ Dominant } & $44(4.2)$ & $42(3.7)$ & 1.00 & 0.58 \\
& AA+AG & & $1016(95.8)$ & $1110(96.3)$ & $0.88(0.57-1.36)$ & \\
\hline & & & & & & \\
\hline
\end{tabular}

Table 5. Haplotype analysis of SNPs in the C3 gene in SCZ patients and controls. ORs, 95\% CIs, and $P$-values were adjusted for age and gender. *Haplotypes with $P>0.05$ or frequency $<1 \%$ in all subjects are not listed

\begin{tabular}{lccccc}
\hline Haplotype* & Total & $\begin{array}{c}\text { Frequency } \\
\text { Case }\end{array}$ & Control & OR $(95 \% \mathrm{CI})$ & $P$ \\
\hline rs11569562-rs344555 & & & & $<0.0001$ \\
CG & 0.5219 & 0.5255 & 0.5189 & 1.00 & --- \\
TG & 0.1949 & 0.1772 & 0.2108 & $0.81(0.69-0.95)$ & 0.012 \\
rs344555-rs2241393 & & & & 0.034 \\
GG & 0.5610 & 0.5591 & 0.5629 & 1.00 & --- \\
AG & 0.0783 & 0.0892 & 0.0680 & $1.29(1.00-1.66)$ & 0.049 \\
rs11569562-rs344555-rs2241393 & & & $<0.0001$ \\
CGG & 0.4646 & 0.4729 & 0.4571 & 1.00 & --- \\
TGG & 0.0977 & 0.0861 & 0.1081 & $0.76(0.60-0.96)$ & 0.019 \\
rs11569562-rs344555-rs2241393-rs2241392-rs11569514 & 0.0058 \\
CGGGA & 0.4089 & 0.3992 & 0.4156 & 1.00 & --- \\
CGGGT & 0.0271 & 0.0370 & 0.0201 & $1.85(1.18-2.91)$ & 0.0073 \\
rs11569514-rs445750-rs451760-rs11672613-rs2230205 & 0.042 \\
ATACA & 0.3243 & 0.3238 & 0.3268 & 1.00 & --- \\
ACGTG & 0.0525 & 0.0599 & 0.0451 & $1.37(1.01-1.85)$ & 0.034 \\
\hline \multicolumn{7}{c}{} \\
\hline
\end{tabular}




\section{Discussion}

In the study, we found no significant difference in the allele frequencies of the 10 examined SNPs in C3 between the cases and controls. However, we did observe a significant difference in the genotype distribution of rs11569514 between SCZ patients and normal controls. Haplotype analyses suggested that there was a combined effect of SNPs in C3 on the risk of developing SCZ.

Over the past decade, variants in several candidate genes have been shown to be associated with SCZ, a polygenic disease. BACE1 variants had a significant association with SCZ in an Iranian population [20]. Weiss's group [17] indicated that several variants of SHANK3 had associations with SCZ. Gender-specific associations between variants of $C H G B$ and SCZ have been revealed in a Korean population by Shin et al. [15]. Conversely, a casecontrol study indicated that there was no significant association between SNPs of FGA and SCZ [19].

The complement system plays a crucial role in innate immunity and also regulates adaptive immune processes [25]. The $C 3$ component of the complement system also plays a central role in the cascade of all three complement activation pathways (alternative, classical, and lectin) [26,27]. While a lack of $C 3$ can contribute to recurrent immune problems, excessive activation of complement proteins is associated with various diseases including Alzheimer's syndrome, Crohn's disease, SCZ, and others [28]. Animal models have demonstrated that the immune system is associated with the development of SCZ [29]. Complex variation of another member of the complement system, C4, has also been shown to be associated with SCZ [30].

The distribution of three types of $C 3$ (F, FS, and S) is different between SCZ patients and healthy controls. The frequency of the C3F gene allele was significantly higher in SCZ patients than in controls [31]. Hakobyan et al. showed that $C 3$ hemolytic activity was 2.01fold higher in SCZ patients than in healthy controls [32]. dos Santos Sória et al. reported that $C 3$ was significant higher in SCZ patients than in control subjects [33]. Nsaiba et al. also demonstrated that C3 was increased in SCZ patients [34].

Although these studies concluded that $C 3$ concentration was significant higher in SCZ patients than in controls [32-34], normal serum levels of $C 3$ in patients with chronic SCZ and lower serum levels of $C 3$ in male Chinese SCZ patients have been reported [35-38]. Therefore, it is necessary to study the relationship between $C 3$ variants and SCZ.

A preliminary investigation on the association of 5 SNPs (rs2277984, rs7951, rs11672613, rs2230205, and rs2230199) in C3 with SCZ found no significant association between these SNPs and SCZ [16]. In our study, we increased the number of subjects and studied the association of 10 tag SNPs (rs11569562, rs344555, rs2241393, rs2241392, rs11569514, rs445750, rs451760, rs11672613, rs2230205, and rs2250656) in C3 with SCZ. We observed a significant difference in the genotype distribution of rs11569514 between the SCZ patients and healthy controls. We also found associations between haplotypes of $C 3$ variants and SCZ.

This study had several limitations. First, the inadequate power of several SNPs may have had an effect on the null association of $C 3$ and SCZ. The range of our sample detection rates was $90.9-99.2 \%$, so some subjects were not included in the genotype analysis of the 10 SNPs, and this might have introduced a selection bias into our study. We studied the association between $C 3$ and SCZ at the genetic level, and further study of the association between $C 3$ and $\mathrm{SCZ}$ at the transcriptional and protein levels is necessary.

In summary, this study tested the association between 10 tag SNPs in C3 and SCZ. To our knowledge, this is the first study to confirm a significant association between $C 3$ variants with SCZ in a northern Chinese Han population. Despite the study limitations, our results may provide useful evidence on the genetic etiology of SCZ. 


\section{Cellular Physiology Cell Physiol Biochem 2018;46:2480-2486 \begin{tabular}{l|l} 
DOI: 10.1159/000489654 & Ond Biochemistry 2018 The Author(s). Published by S. Karger AG, Basel \\
wwww.karger.com/cpb
\end{tabular} \\ Zhang et al.: C3 Gene and Schizophrenia}

\section{Acknowledgements}

This study was supported by grants from the Natural Science Foundation of China (grant no. 81673253) and the Norman Bethune Program of Jilin University (2015227).

\section{Disclosure Statement}

The authors declare that they have no conflict of interests.

\section{References}

Picchioni MM, Murray RM: Schizophrenia. BMJ 2007;335:91-95.

Devor A, Andreassen OA, Wang Y, Maki-Marttunen T, Smeland OB, Fan CC, Schork AJ, Holland D, Thompson WK, Witoelar A, Chen CH, Desikan RS, McEvoy LK, Djurovic S, Greengard P, Svenningsson P, Einevoll GT, Dale AM: Genetic evidence for role of integration of fast and slow neurotransmission in schizophrenia. Mol Psychiatry 2017;22:792-801.

3 Kessler RC, Amminger GP, Aguilar-Gaxiola S, Alonso J, Lee S, Ustun TB: Age of onset of mental disorders: a review of recent literature. Curr Opin Psychiatry 2007;20:359-364.

4 Ochoa S, Usall J, Cobo J, Labad X, Kulkarni J: Gender differences in schizophrenia and first-episode psychosis: a comprehensive literature review. Schizophr Res Treatment 2012;2012:916198.

Harris EC, Barraclough B: Excess mortality of mental disorder. Br J Psychiatry 1998;173:11-53.

Woodberry KA, Giuliano AJ, Seidman LJ: Premorbid IQ in schizophrenia: a meta-analytic review. Am J Psychiatry 2008;165:579-587.

7 Nivard MG, Gage SH, Hottenga JJ, van Beijsterveldt CE, Abdellaoui A, Bartels M, Baselmans BM, Ligthart L, Pourcain BS, Boomsma DI, Munafo MR, Middeldorp CM: Genetic Overlap Between Schizophrenia and Developmental Psychopathology: Longitudinal and Multivariate Polygenic Risk Prediction of Common Psychiatric Traits During Development. Schizophr Bull 2017;43:1197-1207.

-8 Leza JC, Garcia-Bueno B, Bioque M, Arango C, Parellada M, Do K, O’Donnell P, Bernardo M: Inflammation in schizophrenia: A question of balance. Neurosci Biobehav Rev 2015;55:612-626.

-9 Lichtenstein P, Yip BH, Bjork C, Pawitan Y, Cannon TD, Sullivan PF, Hultman CM: Common genetic determinants of schizophrenia and bipolar disorder in Swedish families: a population-based study. Lancet 2009;373:234-239.

10 Nivard MG, Dolan CV, Kendler KS, Kan KJ, Willemsen G, van Beijsterveldt CE, Lindauer RJ, van Beek JH, Geels LM, Bartels M, Middeldorp CM, Boomsma DI: Stability in symptoms of anxiety and depression as a function of genotype and environment: a longitudinal twin study from ages 3 to 63 years. Psychol Med 2015;45:1039-1049.

11 Jones HJ, Stergiakouli E, Tansey KE, Hubbard L, Heron J, Cannon M, Holmans P, Lewis G, Linden DE, Jones PB, Davey Smith G, O’Donovan MC, Owen MJ, Walters JT, Zammit S: Phenotypic Manifestation of Genetic Risk for Schizophrenia During Adolescence in the General Population. JAMA Psychiatry 2016;73:221-228.

-12 Krapohl E, Euesden J, Zabaneh D, Pingault JB, Rimfeld K, von Stumm S, Dale PS, Breen G, O’Reilly PF, Plomin R: Phenome-wide analysis of genome-wide polygenic scores. Mol Psychiatry 2016;21:1188-1193.

13 Schizophrenia Working Group of the Psychiatric Genomics Consortium: Biological insights from 108 schizophrenia-associated genetic loci. Nature 2014;511:421-427.

14 van Os J, Kapur S: Schizophrenia. Lancet 2009;374:635-645.

15 Shin JG, Kim JH, Park CS, Kim BJ, Kim JW, Choi IG, Hwang J, Shin HD, Woo SI: Gender-Specific Associations between CHGB Genetic Variants and Schizophrenia in a Korean Population. Yonsei Med J 2017;58:619-625.

16 Ni J, Hu S, Zhang J, Tang W, Lu W, Zhang C: A Preliminary Genetic Analysis of Complement 3 Gene and Schizophrenia. PLoS One 2015;10:e0136372.

17 de Sena Cortabitarte A, Degenhardt F, Strohmaier J, Lang M, Weiss B, Roeth R, Giegling I, HeilmannHeimbach S, Hofmann A, Rujescu D, Fischer C, Rietschel M, Nothen MM, Rappold GA, Berkel S: Investigation of SHANK3 in schizophrenia. Am J Med Genet B Neuropsychiatr Genet 2017;174:390-398. 


\section{Cellular Physiology Cell Physiol Biochem 2018;46:2480-2486 \begin{tabular}{l|l} 
DOI: 10.1159/000489654 2018 & $\begin{array}{l}\text { O 2018 The Author(s). Published by S. Karger AG, Basel } \\
\text { www.karger.com/cpb }\end{array}$ \\
and Biochemistry Published online: May II,
\end{tabular}}

18 Sudesh R, Priyadarshini T, Preeti R, John S, Thara R, Mowry B, Munirajan AK: Minor allele C of rs12807809 polymorphism in NRGN contributes to the severity of psychosis in patients with Schizophrenia in South Indian population. Neurosci Lett 2017;649:107-111.

19 Rao W, Zhou N, Zhang H, Liu R, Zhang S, Su Y, Yang G, Ma Y, Shi J, Yu Y: A Case-Control Study of the Association between Polymorphisms in the Fibrinogen Alpha Chain Gene and Schizophrenia. Disease Markers 2017;2017:3104180.

20 Rahimi-Aliabadi S, Shahmohammadibeni N, Jamshidi J, Shandiz EE, Mirfakhraie R, Ohadi M, Nejad FR, Mansoori N, Taheri M, Gholipour F, Moudi S, Tayebi G, Divsalar S, Darvish H, Movafagh A: Association of beta-Secretase Functional Polymorphism with Risk of Schizophrenia. Genet Test Mol Biomarkers 2017;21:248-251.

-21 Fananas L, Moral P, Panadero MA, Bertranpetit J: Complement genetic markers in schizophrenia: C3, BF and C6 polymorphisms. Hum Hered 1992;42:162-167.

22 Zhang C, Fang Y, Xie B, Cheng W, Du Y, Wang D, Yu S: DNA methyltransferase 3B gene increases risk of early onset schizophrenia. Neurosci Lett 2009;462:308-311.

23 Zhang W, Tang M, Zhong M, Wang Z, Shang Y, Gong H, Zhang Y, Zhang W: Association of the six transmembrane protein of prostate 2 gene polymorphisms with metabolic syndrome in Han Chinese population. Diabetes Metab Syndr 2013;7:138-142.

24 Sole X, Guino E, Valls J, Iniesta R, Moreno V: SNPStats: a web tool for the analysis of association studies. Bioinformatics 2006;22:1928-1929.

-25 Mayilyan KR, Weinberger DR, Sim RB: The complement system in schizophrenia. Drug News Perspect 2008;21:200-210.

-26 Nauta AJ, Roos A, Daha MR: A regulatory role for complement in innate immunity and autoimmunity. Int Arch Allergy Immunol 2004;134:310-323.

-27 Cole DS, Morgan BP: Beyond lysis: how complement influences cell fate. Clin Sci (Lond) 2003;104:455-466.

-28 Tichaczek-Goska D: Deficiencies and excessive human complement system activation in disorders of multifarious etiology. Adv Clin Exp Med 2012;21:105-114.

29 Patterson PH: Immune involvement in schizophrenia and autism: etiology, pathology and animal models. Behavioural Brain Research 2009;204:313-321.

-30 Aswin S, Bialas AR, Heather DR, Avery D, Hammond TR, Nolan K, Katherine T, Jessy P, Matthew B, Vanessa VD: Schizophrenia risk from complex variation of complement component 4. Nature 2016;530:177-183.

-31 Rudduck C, Beckman L, Franzen G, Lindstrom L: C3 and C6 complement types in schizophrenia. Hum Hered 1985;35:255-258.

32 Hakobyan S, Boyajyan A, Sim RB: Classical pathway complement activity in schizophrenia. Neurosci Lett 2005;374:35-37.

-33 Santos Soria L, Moura Gubert C, Cereser KM, Gama CS, Kapczinski F: Increased serum levels of C3 and C4 in patients with schizophrenia compared to eutymic patients with bipolar disorder and healthy. Rev Bras Psiquiatr 2012;34:119-120.

-34 Nsaiba MJ, Lapointe M, Mabrouk H, Douki W, Gaha L, Perusse L, Bouchard C, Jrad BB, Cianflone K: C3 Polymorphism Influences Circulating Levels of C3, ASP and Lipids in Schizophrenic Patients. Neurochem Res 2015;40:906-914.

-35 Spivak B, Radwan M, Brandon J, Baruch Y, Stawski M, Tyano S, Weizman A: Reduced total complement haemolytic activity in schizophrenic patients. Psychol Med 1993;23:315-318.

-36 Spivak B, Radwan M, Elimelech D, Baruch Y, Avidan G, Tyano S: A study of the complement system in psychiatric patients. Biol Psychiatry 1989;26:640-642.

-37 Wong CT, Tsoi WF, Saha N: Acute phase proteins in male Chinese schizophrenic patients in Singapore. Schizophr Res 1996;22:165-171.

-38 Kano S, Nwulia E, Niwa M, Chen Y, Sawa A, Cascella N: Altered MHC class I expression in dorsolateral prefrontal cortex of nonsmoker patients with schizophrenia. Neurosci Res 2011;71:289-293. 